\title{
Considering Both Health-Promoting and Illness-Related Factors in Assessment of Health-Related Quality of Life After Myocardial Infarction
}

\author{
Eva Brink
}

\author{
Institute of Health and Care Sciences, Sahlgrenska Academy, University of Gothenburg, Sweden \\ Centre for Person-Centred Care (GPCC), University of Gothenburg, Gothenburg, Sweden \\ Department of Nursing, Health and Culture, University West, Trollhättan, Sweden
}

\begin{abstract}
The present study addresses factors that contributed to explaining the variance in health-related quality of life (HRQOL) one year after myocardial infarction, considering both illness-related and health-promoting factors. The aim was to elucidate the influence of depression, fatigue, optimism and sense of coherence on HRQOL. The sample consisted of 95 persons who had been treated for myocardial infarction. Correlation and multiple regression analyses were performed. The results showed that depression, fatigue, optimism and sense of coherence variables were all correlated with both the physical and the mental dimensions of HRQOL. In two multiple regression analyses where the physical component score (PCS) and the mental component score (MCS) were the criterion variables, fatigue contributed to the explained variance in the PCS $(p<0.001)$, and both depression $(p<0.01)$ and fatigue $(p<0.01)$ contributed to explained the variance in the MCS, after controlling for age and gender. To conclude, fatigue was the most significant variable associated with HRQOL, which was interpreted as having the following clinical implication: When a person suffers from post-myocardial infarction fatigue, this must be addressed first. Clearly, it is time to take the problems of patients who suffer from post-myocardial infarction fatigue seriously. It is of vital importance to develop and evaluate fatigue relief strategies in cardiovascular nursing and to test them in interventional studies. This does not exclude investing in healthpromoting factors, e.g. sense of coherence, in coronary care interventions. Intervention strategies focusing on both illnessrelated and salutogentic factors may be optimal. This is a question for further research.
\end{abstract}

Keywords: Health-related quality of life, fatigue, myocardial infarction, salutogenesis.

\section{INTRODUCTION}

Studies have shown that acute myocardial infarction is negatively associated with health-related quality of life (HRQOL) $[1,2]$ and that persons with myocardial infarction score lower on HRQOL compared with persons with a variety of other diseases, with the exception of stroke [3]. HRQOL is a concept intended to mirror health experiences and to cover what people are able to do (functioning), how they feel (distress and well-being), and how they evaluate their state of health (personal evaluation). Physical and mental health are principal components of health-related quality of life [4].

In analyses of HRQOL after myocardial infarction, there are two alternative foci from which to select: a) a healthpromoting/salutogenetic perspective and b) an illnessrelated/pathogenetic perspective. In a salutogenetic perspective, the focus is on factors that promote health and well-being [5]. One such factor is sense of coherence, and research has shown that this coping resource may promote quality of life in people with chronic disease [6]. Sense of coherence (SOC) mirrors a global orientation that expresses the extent to which a person is confident that demands (inner

*Address correspondence to this author at the Institute of Health and Care Sciences, The Sahlgrenska Academy at University of Gothenburg, Box 457, SE 40530 Gothenburg, Sweden; Tel: +46 31 7866104;

E-mail: eva.brink@gu.se and outer) in the course of living are structured, predictable and explicable as well as the extent to which the person feels he/she has the resources to meet demands worthy of engagement [7]. In general, the stronger one's SOC, the better one's perceived health, especially with regard to mental health [8]. Persons recovering from first-time myocardial infarction with high scores on SOC were found to have fewer angina attacks, were more physically active and more satisfied with treatment [9]. In addition, SOC has been found to be positively related to quality of life after myocardial infarction in women [10]. People also differ widely in life orientation as regards optimism and pessimism. Optimism has been defined in terms of positive outcome expectancies, and it is assumed that optimism serves as a protective function in the face of life's difficulties and health threats [11]. Positive well-being, including optimism, was associated with a reduced risk for coronary heart disease [12] and lowered rehospitalization rates after coronary artery surgery [13]. Optimism has not been studied in relation to HRQOL after myocardial infarction.

In a pathogenetic perspective, risk factors associated with negative illness experiences are investigated. Fatigue and depression are risk factors associated with reduced HRQOL $[1,2]$. Approximately one-third of patients report some degree of depression one year after myocardial infarction [14]. Gender differences in depressive symptoms, as they relate to HRQOL, have been identified, with women scoring more depression and poorer physical and mental health 
compared to men [15]. Moreover, as many as 50 percent of patients have been found to report fatigue four months after a myocardial infarction [16]. Experiences of illness-related fatigue include feelings of tiredness, weakness and lack of energy, and consequently, fatigue is closely related to depression after myocardial infarction [17]. Depressed people primarily experience fatigue, as fatigue is a key symptom of depression [18]. However, only one-third of those experiencing fatigue during early recovery from myocardial infarction reported possible depression [16], meaning that fatigue can be a distressing symptom with or without coexisting depression [19].

In the present study, the research question addresses which factors best explain the variance in HRQOL after myocardial infarction, considering both illness-related and health-promoting factors. The aim was to elucidate the influence of depression, fatigue, optimism and sense of coherence on HRQOL.

\section{MATERIALS AND METHODOLOGY}

\section{Participants}

The sample consisted of 98 persons who had been treated for myocardial infarction in a coronary care unit at a Swedish rural hospital and who were participating in a longitudinal study of health-related quality of life after myocardial infarction. We have previously reported longitudinal results identifying illness-related predictors of HRQOL measured five days and five months after myocardial infarction [1]. The present study deals with cross-sectional data from the 1-year follow-up and investigates the influence of both illness-related and salutogenetic factors on HRQOL. Correlation analyses were performed to explore relationships between variables, and two multiple regression models were tested to identify the cross-sectional variables that best explained the variance in the physical dimension and the mental dimension of HRQOL one year after myocardial infarction.

\section{Instruments}

The Short Form 36 (SF-36) is a generic HRQOL questionnaire that groups items into eight subscales. It also provides a summary of physical and mental measures, the physical component score (PCS) and the mental component score (MCS), which were used in the present study [4]. Cronbach's $\alpha$ for both PCS and MCS were 0.91. The Sense of Coherence (SOC) Scale refers to a global orientation that is assumed to indicate ability to maintain health [7]. The short 13-item version was used in the present study. Each item of the SOC has a 7-point response format. A high summed score indicates high SOC and Cronbach's $\alpha$ for the scale was 0.82 . Optimism-Life orientation Test (LOT) was developed by to measure optimism [11]. The LOT includes eight items rated on a 5-point scale, ranging from (0) strongly disagree to (4) strongly agree. A high scale score indicates an optimistic life orientation. In the present study, Cronbach's $\alpha$ for the scale was 0.79 . Fatigue was measured using the Somatic Health Complaints Questionnaire (SHCQ), which addresses common health problems in cardiac patients [20]. In the present study, the subscale fatigue - consisting of items about general fatigue, weakness, lack of energy, and dizziness - was used. Each item has a 6-point response scale ranging from (1) never to (6) always. A high scale score indicates fatigue. In the present study, Cronbach's $\alpha$ for the scale was 0.85 . Depression was measured using the Hospital Anxiety Depression Scale (HADS), which consists of two subscales: one for anxiety and one for depression. The depression scale was used here. Each item has a 4-point response scale ranging from (0) not at all to (3) mostly. Scale scores of $\geq 8$ point to possible depression, and scores $\geq 11$ indicate probable depression [21]. In the present study, Cronbach's $\alpha$ for the scale was 0.81 .

\section{Data Analysis}

Descriptive statistics comprised mean scores and standard deviations. Gender differences were tested using an independent sample $t$-test. Person's correlations coefficients were used to identify associations between variables. Thereafter, two multiple regression models were employed for prediction of HRQOL after one year: one for PSC and the other for MCS. The criterion variables were depression, fatigue, optimism and sense of coherence. Age and gender were controlled for. Data were analysed using SPSS, version 17.0. The level of significance was set at $5 \%$.

\section{RESULTS}

The sample consisted of 98 persons, 33 women and 65 men with a mean age of 67.9 years. The women were on average 6.9 years older than the men $(p<0.01)$. The participants had been treated for myocardial infarction in a coronary care unit at a Swedish rural hospital one year

Table 1. Mean Scores (SD) for SF-36 Dimensions, Depression, Fatigue, Optimism, SOC, and Age for the Total Group and with Gender Comparisons $(n=98)$

\begin{tabular}{|c|c|c|c|c|}
\hline Variable & Total & Women & Men & p \\
\hline \hline SF-36; PCS & $42.21(11.20)$ & $37.45(11.56)$ & $44.63(10.27)$ & $<0.01$ \\
\hline SF-36; MCS & $48.16(11.13)$ & $47.66(12.20)$ & $3.41(10.64)$ & n.s. \\
\hline Depression & $3.22(3.24)$ & $3.46(3.55)$ & $9.90(4.40)$ & n.s. \\
\hline Fatigue & $10.09(4.34)$ & $10.48(4.27)$ & $16.52(3.45)$ & n.s. \\
\hline Optimism & $16.68(3.53)$ & $17.00(3.72)$ & $74.42(10.27)$ & n.s. \\
\hline SOC & $74.14(10.09)$ & $73.61(9.86)$ & $65.58(9.82)$ & $<0.01$ \\
\hline Age & $67.89(9.95)$ & $72.45(8.66)$ & & \multicolumn{2}{c|}{} \\
\hline
\end{tabular}


Table 2. Zero-Order Correlations

\begin{tabular}{|c|c|c|c|c|c|c|}
\hline Variables & PCS & MCS & Depression & Fatigue & Optimism & SOC \\
\hline PCS & - & & & & & \\
\hline Depression & $-0.407 * * *$ & $-0.662 * * *$ & - & & & \\
\hline Fatigue & $-0.562 * * *$ & $-0.626 * * *$ & $0.636 * * *$ & - & & \\
\hline SOC & $0.315 * *$ & $0.315^{* *}$ & $-0.486^{* * *}$ & $-0.321 * *$ & $0.297 * *$ & - \\
\hline
\end{tabular}

Note: $\mathrm{PCS}=$ physical component score; $\mathrm{MCS}=$ mental component score; $\mathrm{SOC}=$ sense of coherence.

$* P<0.05 . * * P<0.01 . * * * P<0.001$

earlier. Initially, the normality of the distribution scores for HRQL for the sample as a whole was assessed. Inspection of the normal probability plots (Normal Q-Q Plots) and the boxplots indicated normality, and the 5\% Trimmed means for PCS and MCS were close to the original means, meaning that the two distributions of scores were sufficiently normal. Descriptive data with gender comparisons for HRQOL, depression, fatigue, optimism and sense of coherence are presented in Table 1. One gender difference was that the women scored lower on the physical dimension (PCS) of HRQOL than the men did $(p<0.01)$.

The four independent variables - depression, fatigue, optimism and sense of coherence - were all correlated with both the physical and the mental dimensions of HRQOL (Table 2). Consequently, the two multiple regression models for prediction of PCS and MCS included fatigue, sense of coherence and optimism, controlling for age and gender. The analyses showed that the explained variance of PCS was $45.2 \%, F(6,90)=14.30$ with fatigue functioning as a significant predictor $(\mathrm{p}<0.001)$. The explained variance of MCS was $50.5 \%, \mathrm{~F}(6,90)=17.63$, with depression $(\mathrm{p}<$ $0.01)$ and fatigue $(\mathrm{p}<0.01)$ functioning as predictors (Table 3).

\section{DISCUSSION}

In the present study, which considered both healthpromoting and illness-related factors in assessment of HRQOL after myocardial infarction, fatigue turned out to be the most significant factor influencing self-reported HRQOL. In the regression models, fatigue explained the variance in the PCS and, both fatigue and depression contributed to the variance in the MCS. Optimism and sense of coherence did not contribute to significant variation in mental and physical HRQOL. If the intention of coronary care is to optimize patients' HRQOL, the present findings imply that post-myocardial infarction fatigue and depression need to be emphasized. Such a priority does not indicate that sense of coherence or optimism are negligible factors in promoting HRQOL after myocardial infarction, but it does mean that, as a first step, a depressed person may need professional support and treatment and a fatigued person may need symptom relief support.

In general, sense of coherence is clearly a health resource that affects quality of life [6]. Sense of coherence has been found to predict HRQOL after myocardial infarction, but after controlling for disease severity the association with PCS disappeared [22]. This finding gives some support for the result of the present study; that symptom burden has to be considered. According to a recent study sense of coherence can change over time after myocardial infarction and physical limitations could be a factor that restrains positive change [9]. Moreover, research has shown that physical functioning decreases after myocardial infarction [23]. Fatigue may be an indicator of decreased physical functioning, and is therefore an important factor to consider in alleviation of physical limitations, and consequently in strengthening a person's sense of coherence. Consequently, it is likely that an intervention directed at supporting fatigue relief self-care strategies, as well as at strengthening a person's sense of coherence, will have positive consequences for HRQOL. A combination of health-promoting and illness-

Table 3. Multiple Linear Regressions of Health-Related Quality of Life; the Physical Dimension Score, PCS and the Mental Dimension Score, MCS $(n=98)$

\begin{tabular}{|c|c|c|c|c|c|c|c|c|}
\hline Variable & \multicolumn{4}{|c|}{$\operatorname{PCS}\left(\mathbf{R}_{\text {adj }}^{2}=0.452\right)$} & \multicolumn{4}{|c|}{$\operatorname{MCS}\left(R_{\text {adj }}^{2}=0.505\right)$} \\
\hline Fatigue & -4.405 & 1.045 & -0.430 & $<0.001$ & -3.459 & 0.995 & -.0337 & 0.001 \\
\hline Optimism & -0.219 & 0.304 & -0.069 & 0.474 & 0.242 & 0.289 & 0.076 & 0.406 \\
\hline Age & -0.334 & 0.091 & -0.297 & $<0.001$ & 0.179 & 0.087 & 0.159 & 0.042 \\
\hline Gender & 4.287 & 1.892 & 0.183 & 0.026 & 2.317 & 1.802 & 0.099 & 0.202 \\
\hline
\end{tabular}


related intervention strategies in contemporary coronary rehabilitation may be optimal, but this is a question for further research.

In some studies, optimism in the form of positive outcome expectancies has been associated with reduced likelihood of reporting distressing symptoms [11, 24]. However, de Ridder et al. did not confirm a general relationship between optimism and symptom report in patients with multiple sclerosis, but found that positive outcome expectancies contributed to less severe reported fatigue symptoms in patients with Type 1 diabetes mellitus, which was interpreted either as an underestimation of symptoms or as being due to adequate fatigue coping strategies [25]. In the present sample, optimism was negatively correlated (zero-order correlation) with depression and fatigue, but in the multiple regression analyses optimism did not contribute significantly to explaining the variance in HRQOL. One possible interpretation of these findings is that post-myocardial infarction fatigue serves as a disruptive force, preventing patients from maintaining daily activities and impacting negatively on HRQOL. Consequently, when a person cannot cope with the demands of daily life due to lack of energy, an optimistic life orientation is not sufficient. In an earlier analysis of associations between optimism and fatigue [26], we discussed the non-linear relationship between variables and suggested that a pessimistic view of life may have more negative influences on post-myocardial infarction fatigue than an optimistic view has positive influences. In addition, a study identified a zero-order correlation between optimism and quality of life in patients treated for coronary heart disease, but when variables such as control appraisals and specific expectancies were included in the model, optimism no longer predicted quality of life [27].

The independent variables included in the multiple regression models in the present study were significantly correlated, and therefore, multicollinearity was considered. Multicollinearity does not reduce the predictive power or reliability of the regression model as a whole, but it may not give valid results for any single independent variable. However, all bivariate correlation coefficients were less than 0.7 , and the SPSS collinearity statistics (Tolerance value and Variance inflation factor) did not indicate multicollinearity; thus all variables were retained.

One limitation of the present study is that the results are based on a small sample. With a larger sample size, the influence of health-promoting variables on HRQOL could possibly have reached significant levels. On the other hand, the finding that fatigue was a significant factor influencing both the mental and the physical dimension of HRQOL would seem to be important to emphasize. The suggestion that fatigue should be considered more fully in coronary rehabilitation also finds support in results from previous research [19, 28, 29].

\section{CONCLUSION}

One year after myocardial infarction, illness-related factors were more strongly associated with HRQOL than were health-promoting factors. Fatigue was a significant predictor of HRQOL, which was interpreted as having the following clinical implication: When a person cannot cope with the demands of daily life due to fatigue, this must be addressed first. Clearly, it is time to take the problems of patients who suffer from post-myocardial infarction fatigue seriously. It is of vital importance to develop and evaluate fatigue relief strategies in cardiovascular nursing and to test them in intervention studies. This does not exclude investing in health-promoting factors, e.g. sense of coherence, in coronary care interventions. Interventional strategies focusing on both illness-related and salutogentic factors may be optimal. This is a question for further research.

\section{ACKNOWLEDGEMENT}

Declared none.

\section{CONFLICT OF INTEREST}

\author{
Declared none.
}

\section{REFERENCES}

[1] Brink E, Grankvist G, Karlson BW, Hallberg LR. Health-related quality of life in women and men one year after acute myocardial infarction. Qual Life Res 2005; 14(3): 749-57

[2] de Jonge P, Spijkerman TA, van den Brink RH, Ormel J. Depression after myocardial infarction is a risk factor for declining health related quality of life and increased disability and cardiac complaints at 12 months. Heart 2006; 92(1): 32-9.

[3] Bach JP, Riedel O, Pieper L, Klotsche J, Dodel R, Wittchen HU. Health-related quality of life in patients with a history of myocardial infarction and stroke. Cerebrovasc Dis 2011;31(1): 6876.

[4] Ware JE, Kosinski M, Keller SD. SF-36 physical and mental health summary scales - a user's manual. Boston MA: New England Medical Center 1994.

[5] Lindström B, Eriksson M. The hitchhiker's guide to salutogenesis: Salutogenic pathways to health promotion. Helsinki: Tuokinprint Oy 2010.

[6] Eriksson M, Lindstrom B. Antonovsky's sense of coherence scale and its relation with quality of life: a systematic review. J Epidemiol Community Health 2007; 61(11): 938-44.

[7] Antonovsky A. The structure and properties of the sense of coherence scale. Soc Sci Med 1993; 36(6): 725-33.

[8] Eriksson M, Lindstrom B. Antonovsky's sense of coherence scale and the relation with health: a systematic review. J Epidemiol Community Health 2006 May; 60(5): 376-81.

[9] Bergman E, Malm D, Bertero C, Karlsson JE. Does one's sense of coherence change after an acute myocardial infarction? A two-year longitudinal study in Sweden. Nurs Health Sci 2011; 13(2): 156-63.

[10] Norekval TM, Fridlund B, Moons P, et al. Sense of coherence - a determinant of quality of life over time in older female acute myocardial infarction survivors. J Clin Nurs 2010; 19(5-6): 820-31.

[11] Scheier MF, Carver CS. Optimism, coping, and health: assessment and implications of generalized outcome expectancies. Health Psychol 1985; 4: 219-47.

[12] Boehm JK, Kivimaki M, Peterson C, Kubzansky L. A Prospective Study of Positive Psychological Well-Being and Coronary Heart Disease. Health Psychol 2011; 30(3): 259-67.

[13] Scheier MF, Matthews KA, Owens JF, et al. Optimism and rehospitalization after coronary artery bypass graft surgery. Arch Intern Med 1999; 159(8): 829-35.

[14] Frasure-Smith N, Lesperance F, Gravel G, et al. Social support, depression, and mortality during the first year after myocardial infarction. Circulation 2000; 101(16): 1919-24.

[15] Norris CM, Hegadoren K, Pilote L. Depression symptoms have a greater impact on the 1-year health-related quality of life outcomes of women post-myocardial infarction compared to men. Eur $\mathbf{J}$ Cardiovasc Nurs 2007; 6(2): 92-8.

[16] Alsen P, Brink E, Brandstrom Y, Karlson BW, Persson LO. Fatigue after myocardial infarction: relationships with indices of emotional distress, and sociodemographic and clinical variables. Int J Nurs Pract 2010; 16(4): 326-34.

[17] Fennessy MM, Fink AM, Eckhardt AL, et al. Gender differences in fatigue associated with acute myocardial infarction. J Cardiopulm Rehabil Prev 2010; 30(4): 224-30. 
[18] Diagnostic and statistical manual of mental disorders: DSM-IV-TR. Washington, DC: American Psychiatric Association 2000.

[19] McGowan L, Dickens C, Percival C, Douglas J, Tomenson B, Creed F. The relationship between vital exhaustion, depression and comorbid illnesses in patients following first myocardial infarction. J Psychosom Res 2004; 57(2): 183-8.

[20] Brink E, Cliffordson C, Herlitz J, Karlson BW. Dimensions of the Somatic Health Complaints Questionnaire (SHCQ) in a sample of myocardial infarction patients. Eur J Cardiovasc Nurs 2007; 6(1): 27-31.

[21] Zigmond AS, Snaith RP. The hospital anxiety and depression scale. Acta Psychiatr Scand 1983; 67(6): 361-70.

[22] Silarova B, Nagyova I, Rosenberger J, et al. Sense of coherence as an independent predictor of health-related quality of life among coronary heart disease patients. Qual Life Res 2012 [Epub ehead of print].

[23] Failde, II, Soto MM. Changes in Health Related Quality of Life 3 months after an acute coronary syndrome. BMC Public Health 2006; 6: 18 .
[24] Andersson G. The benefits of optimism: a meta-analytic review of the life orientation test. Pers Individ Dif 1996; 21(5): 719-25.

[25] de Ridder D, Fournier M, Bensing J. Does optimism affect symptom report in chronic disease? What are its consequences for self-care behaviour and physical functioning? J Psychosom Res 2004; 56(3): 341-50.

[26] Brink E, Grankvist G. Associations between depression, fatigue, and life orientation in myocardial infarction patients. J Cardiovasc Nurs. 2006; 21(5): 407-11

[27] Fitzgerald TE, Tennen H, Affleck G, Pransky GS. The relative importance of dispositional optimism and control appraisals in quality of life after coronary artery bypass surgery. J Behav Med 1993; 16(1): 25-43.

[28] Alsen P, Brink E, Persson LO. Living with incomprehensible fatigue after recent myocardial infarction. J Adv Nurs 2008; 64(5): $459-68$.

[29] Appels A. Exhaustion and coronary heart disease: the history of a scientific quest. Patient Educ Couns 2004; 55(2): 223-9. 